\title{
Polyakov linear- $\sigma$ model in mean-field approximation and optimized perturbation theory
}

\author{
Abdel Nasser Tawfik (1) \\ Nile University, Egyptian Center for Theoretical Physics (ECTP), Juhayna Square of 26th-July-Corridor, 12588 Giza, Egypt \\ and Goethe University, Institute for Theoretical Physics (ITP), Max-von-Laue-Str. 1, D-60438 Frankfurt am Main, Germany \\ Carsten Greiner \\ Goethe University, Institute for Theoretical Physics (ITP), Max-von-Laue-Str. 1, D-60438 Frankfurt am Main, Germany
}

Abdel Magied Diab (i)

Modern University for Technology and Information (MTI), 11571 Cairo, Egypt

and World Laboratory for Cosmology And Particle Physics (WLCAPP), 11571 Cairo, Egypt

M. T. Ghoneim and H. Anwer

Physics Department, Faculty of Science, Cairo University, 12613 Giza, Egypt

(Received 21 August 2019; accepted 10 February 2020; published 30 March 2020)

\begin{abstract}
The optimized perturbation theory (OPT) is confronted to first-principle lattice simulations. We compare results from the Polyakov linear-sigma model (PLSM) in OPT with the conventional mean-field approximation (MFA). At finite temperatures and chemical potentials, the chiral condensates and the decofinement order parameters, the thermodynamic pressure, the pseudocritical temperatures, the subtracted condensates, the second- and high-order moments of various conserved charges (cumulants) obtained in MFA are compared with OPT and also confronted to available lattice quantum chromodynamics (QCD) simulations. We conclude that when moving from lower- to higher-order moments of different quantum charges, OPT becomes more closer to lattice QCD simulations. The higher-order moments of conserved charges, such as baryon, strange, and electric charge, are proportional to powers of the correlation length and thus expected to diverge at the critical endpoint of the QCD phase boundary. Making sure that one approximate approach is more sensitive than another, even slightly, is crucial for implementing PLSM, for instance, in positioning critical endpoint and providing an important signature for the possible experimental detection.
\end{abstract}

DOI: 10.1103/PhysRevC.101.035210

\section{INTRODUCTION}

In quantized field theory [1-6], the linear- $\sigma$ model [7] (LSM) with a spinless scalar field $\sigma_{a}$ [8] and triplet pseudoscalar fields $\pi_{a}$ was introduced in order to describe the pion-nucleon interactions and the chiral degrees of freedom. This low-energy effective model has the generators $T_{a}=\lambda_{a} / 2$, where $\lambda_{a}$ are Gell-Mann matrices, and a real classical field forming an $O(4)$ vector; $\vec{\Phi}=T_{a}\left(\vec{\sigma}_{a}, i \vec{\pi}_{a}\right)$. The chiral symmetry is explicitly broken by the $3 \times 3$ matrix field $H=T_{a} h_{a}$, where $h_{a}$ are the external fields. Under $\mathrm{SU}(2)_{L} \times$ $\mathrm{SU}(2)_{R}$ chiral transformation, for instance, $\Phi \rightarrow L^{+} \Phi R, \sigma_{a}$ acquires finite vacuum expectation values, which in turn break

*atawfik@nu.edu.eg

Published by the American Physical Society under the terms of the Creative Commons Attribution 4.0 International license. Further distribution of this work must maintain attribution to the author(s) and the published article's title, journal citation, and DOI. Funded by $S C O A P^{3}$.
$\mathrm{SU}(2)_{L} \times \mathrm{SU}(2)_{R}$ down to $\mathrm{SU}(2)_{L+R}$. This results in massive $\sigma$ particle and light or even nearly massless Goldstone bosons. Accordingly, the constituent quarks gain masses, as well, $m_{q}=g f_{\pi}$, where $g$ is the coupling and $f_{\pi}$ is the pion decay constant. In the same matter, fermions can be inserted in this model either as nucleons or as quarks. It has been shown that the $\sigma$ field under chiral transformations exhibits the same behavior as that of the quark condensates, i.e., $\sigma$ can be taken as an order parameter for the quantum chromodynamics (QCD) chiral phase transition [9-13]. Accordingly, the phase structure $[12,14-16]$, the properties of QCD in finite magnetic fields [16-19], and also various thermodynamic quantities have been estimated and reported, at finite baryon density $[14,18,20]$ and isospin asymmetry [21]. In almost all these studies, a comprehensive confrontation with the first-principle lattice calculations was the main part. The LSM with its various extensions as examples on QCD-like effective models put forward a major goal to-as perfect as possiblereproduce various quantities simulated in lattice QCD.

Quantum mechanically, the spontaneous symmetry breaking could be achieved by introducing a coherent state and minimizing the free energy density. Assuming that the system 
of interest is enclosed in a cubic box and imposing periodic boundary conditions, the fields can be uniquely decomposed [22] into $\phi(\mathbf{r}, t)=\langle\phi(t)\rangle+\delta \phi(\mathbf{r}, t)=\sum_{k} \phi_{k}(t) \exp (i k \cdot \mathbf{r})$, where $\langle\phi(t)\rangle$ is the spacial expectation value over the box volume and $\delta \phi(\mathbf{r}, t)$ stands for the remaining fluctuations relative to a constant background field. It is obvious that $\phi_{k}=$ $\langle\phi \exp (-i k \cdot \mathbf{r})\rangle$ and the Fourier coefficients satisfy the symmetry relation, $\phi_{k}^{*}=\phi_{-k}$ because the fields, themselves, are real. Having all these estimated, we can now apply the meanfield approximation (MFA), which is originated in statistical physics. In the LSM, the meson fields (or fermion/quark fields) are replaced by their spacial averaged values and all vacuum and thermal fluctuations are neglected. All quarks and antiquarks are retained as quantum fields, but averaged as well.

A generalization of MFA could be the optimized perturbation theory (OPT), also known as $\delta$ expansion or variational perturbation theory [23]. OPT was developed in $O(N) \phi^{4}$ theory and resums higher-order terms of the naive perturbation approach [24,25]. Implications in the symmetric and broken phases [26], Bose-Einstein condensation - Bardeen Cooper Schrieffer theory of superconductivity (BEC-BCS) [27], and zero-dimensional $O(N)$ scalar field model [28], are examples of the reliability and applicability of OPT. Here, we apply OPT to $O(4) \sigma$ model and then compare the results with the ones obtained from MFA. We aim at determining the sensitivity of OPT relative to MFA. This is the first time, where both approaches are compared with each other with reference to the first-principle lattice calculations. Assuring that one approximate approach is more sensitive than another, even slightly, is very crucial for the preparation of the Polyakov linear- $\sigma$ model (PLSM) for a comprehensive implementation in mapping out the QCD critical boundary and eventually finding the critical endpoint (CEP) and thus providing an crucial signature for the possible experimental detection.

The present paper is organized as follows. A short review on the optimized perturbation theory and the mean-field approximation will be given in Sec. II. Section III is devoted to the results and discussion. This includes chiral condensates and deconfinement order parameters in Sec. III A, pseudocritical temperatures in Sec. III B, thermodynamic pressure in Sec. III C, fluctuations and correlations of conserved charges, in Sec. III D. The latter are detailed to second-order in Sec. III D 1, and higher-order moments in Sec. III D 2. The final conclusions are outlined in Sec. IV.

\section{OPTIMIZED PERTURBATION THEORY AND MEAN-FIELD APPROXIMATION}

We intend to check whether the optimized perturbation theory (OPT) would be able to play the role of an alternative to the nonperturbative approximation, such as the mean-field approximation (MFA), of the PLSM. This is the primary goal set forward for the present paper. In general, an OPT procedure aims at optimizing a linear $\delta$ expansion to the Lagrangian density. The basic idea of OPT becomes obvious when expanding the chiral Lagrangian, Eq. (1), from which we realize that even analytic nonperturbative calculations beyond what MFA would reach become accessible. Concretely, the OPT procedure in PLSM goes as follows:

$$
\mathcal{L}^{\delta}=(1-\delta) \mathcal{L}_{0}(\eta)+\delta \mathcal{L}=\mathcal{L}_{0}(\eta)+\delta\left[\mathcal{L}-\mathcal{L}_{0}(\eta)\right],
$$

where $\eta$ is an arbitrary mass parameter, which can be fixed through an appropriate variational method [29], ${ }^{1}$ and $\mathcal{L}_{0}(\eta)$ is the free Lagrangian density, in which $\eta$ is included. The parameter $\eta$ is equivalent to mass. Thus, the implementation of OPT to PLSM is apparently accompanied by an expansion in terms of the arbitrary parameter $\delta$. Accordingly, Eq. (1) shows that the underlying symmetries in the chiral limit seems to not be modified. The term which is proportional to $\eta$ is added to the Lagrangian, while the same term multiplied by $\delta$ is then subtracted. At $\delta=1$, the original Lagrangian $\mathcal{L}$ can be recovered, straightforwardly. At $\delta=0$, a solvable Lagrangian $\mathcal{L}_{0}$ is obtained. In general, the parameter $\delta$ is also used as a dummy constant, in order to label the orders of the perturbative calculations. Thus, $\delta$ is initially taken as a small value and afterwards fixed to unity.

A short review on the LSM is now in order. LSM has chiral Lagrangian of $N_{f}$ quark flavors including the structure of mesons and quarks. With the incorporation of the Polyakovloop potential,

$$
\mathcal{L}=\mathcal{L}_{\bar{\psi} \psi}+\mathcal{L}_{m}-\mathcal{U}\left(\phi, \phi^{*}, T\right),
$$

where the first term stands for the Lagrangian density for baryonic (fermionic) fields with $N_{c}$ color degrees of freedom, the second term gives the contributions of the mesonic (bosonic) fields, and finally the third term represents the Polyakov-loops potential incorporating the gluonic degrees of freedom and the dynamics of the quark-gluon interactions.

When implementing the OPT approach, Eq. (1), on the PLSM Lagrangian, Eq. (2), we get

$$
\begin{aligned}
\mathcal{L}_{\bar{\psi} \psi}= & \sum_{f} \bar{\psi}_{f}\left[i \gamma^{\mu} D_{\mu}-\delta g T_{a}\left(\sigma_{a}+i \gamma_{5} \pi_{a}\right)\right. \\
& -(1-\delta) \eta] \psi_{f}, \\
\mathcal{L}_{m}= & \operatorname{Tr}\left[\partial_{\mu} \Phi^{\dagger} \partial^{\mu} \Phi-\left(m^{2}+(1-\delta) \eta^{2}\right) \Phi^{\dagger} \Phi\right] \\
& +\delta\left\{c\left(\operatorname{Det}[\Phi]+\operatorname{Det}\left[\Phi^{\dagger}\right]\right)-\lambda_{1}\left(\operatorname{Tr}\left[\Phi^{\dagger} \Phi\right]\right)^{2}\right. \\
& \left.-\lambda_{2} \operatorname{Tr}\left[\Phi^{\dagger} \Phi\right]^{2}+\operatorname{Tr}\left[H\left(\Phi+\Phi^{\dagger}\right)\right]\right\} \\
\mathcal{U}\left(\phi, \phi^{*}, T\right)= & -b T\left[54 \phi \phi^{*} e^{-a / T}+\ln \left(1-6 \phi \phi^{*}\right.\right. \\
& \left.\left.-3\left(\phi \phi^{*}\right)^{2}+4\left(\phi^{3}+\phi^{* 3}\right)\right)\right],
\end{aligned}
$$

where $\psi$ are Dirac spinor fields for the quark flavors $f=$ $[u, d, s]$, while $D_{\mu}, \mu, \gamma^{\mu}$, and $g$ are covariant derivative, Lorentz index, chiral spinors, and Yukawa coupling constant, respectively. $\phi$ and $\phi^{*}$ are the order parameter of the Polyakov-loop variables and their conjugates, respectively. We notice that that Polyakov Lagrangian, Eq. (5), is not directly impacted by the OPT approach, for instance, $\eta$ or $\delta$ are not present, while $\mathcal{L}_{\bar{\psi} \psi}$ and $\mathcal{L}_{m}$ are impacted. We also notice that $\mathcal{L}_{\bar{\psi} \psi}$ is given in terms of $\eta$, while $\mathcal{L}_{m}$ of $\eta^{2}$. The reason for this can be understood due the OPT approach and the role

\footnotetext{
${ }^{1}$ This parameter is different from the PLSM parameters.
} 
of $\eta$, Eq. (1). It is obvious that $\eta$ as a modified mass parameter goes with the masses in the PLSM. Last but not least, when $\delta \rightarrow 0$, the standard PLSM Lagrangian [18] can be obtained. Up to now, construction of PLSM Lagrangian, MFA does not apply.

It should be noticed that the Lagrangian density of the fermions is deformed by adding a gaussian term $\bar{\psi}(1-\delta) \eta \psi$ to the original Lagrangian density. All terms, in which the coupling constant $g$ is included, are multiplied by $\delta$. As discussed earlier, at $\delta \rightarrow 1$, the original Lagrangian density of the fermionic and mesonic contributions can entirely be recovered. The generator operator $\Phi$ is a complex matrix for nonet meson states, $\Phi=\sum_{a=0}^{N_{f}^{2}-1} T_{a}\left(\bar{\sigma}_{a}+\mathrm{i} \bar{\pi}_{a}\right)$. In U(3) algebra, the generator operators $T_{a}=\hat{\lambda}_{a} / 2$ are related to the Gell-Mann matrices $\hat{\lambda}_{a}$ [30]. The parameters $m^{2}, h_{l}, h_{s}, \lambda_{1}, \lambda_{2}$, and $c$ are determined, at $\sigma$ meson $m_{\sigma}=800 \mathrm{MeV}$ [31]. It should be emphasized that the estimation of all these parameters is not affected by MFA or OPT approaches. They are depending on masses and decay constants as partly elaborated in the well-known PCAC relations [31,32].

When using OPT instead of MFA to evaluate the free energy $\mathcal{F}$ of the PLSM, analytic nonperturbative calculations become possible through a prescription known as the principle of minimal sensitivity (PMS) [33,34]. PMS states that $\mathcal{F}$, Eq. (9), can be minimized to the variations of $\eta$, at $\delta=1$ :

$$
\left.\frac{\partial \mathcal{F}_{\mathrm{OPT}}}{\partial \eta}\right|_{\bar{\eta}, \delta=1}=0 .
$$

Accordingly, we emphasize that the expectation value of $\bar{\eta}$ is related to the sigma fields $\sigma_{f} ; \eta \sim \sigma_{f}$ [35]. Consequently, the grand-canonical partition function ${ }^{2} \mathcal{Z}$, which is given in dependence on the temperature $T$ and the chemical potentials of $f$ th quark flavor $\mu_{f}$ is defined by the path integral over all bosons, fermions, antifermions:

$$
\begin{aligned}
\mathcal{Z}= & \operatorname{Tr} \exp \left[\frac{\sum_{f=u, d, s} \mu_{f} \hat{\mathcal{N}}_{f}-\hat{\mathcal{H}}}{T}\right] \\
= & \int \prod_{a} \mathcal{D} \sigma_{a} \mathcal{D} \pi_{a} \int \mathcal{D} \psi \mathcal{D} \bar{\psi} \\
& \times \exp \left[\int d^{4} x\left(\mathcal{L}+\sum_{f=u, d, s} \mu_{f} \bar{\psi}_{f} \gamma^{0} \psi_{f}\right)\right],
\end{aligned}
$$

where $\hat{\mathcal{H}}$ the chiral Hamiltonian density. The chemical potentials $\mu_{f}$ are related to the conserved charge numbers of baryon number $(B)$, electric charge $(Q)$, and strangeness $(S)$ for each of the quark flavors, $f=[u, d, s]$ :

$$
\begin{aligned}
& \mu_{u}=\frac{\mu_{B}}{3}+\frac{2 \mu_{Q}}{3}, \\
& \mu_{d}=\frac{\mu_{B}}{3}-\frac{\mu_{Q}}{3}, \\
& \mu_{s}=\frac{\mu_{B}}{3}-\frac{\mu_{Q}}{3}-\mu_{S} .
\end{aligned}
$$

\footnotetext{
${ }^{2}$ MFA also enters the play but only when constructing the partition function $\mathcal{Z}$ as detailed in Refs. [12,14-21,36].
}

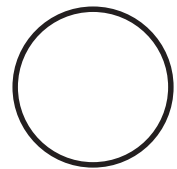

(a)

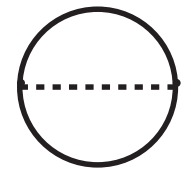

(b)

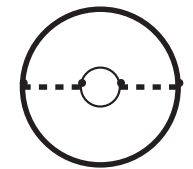

(c)
FIG. 1. Diagrams illustrating the corrections to the free energy up to $\delta^{2}$ expansion. The fermionic contributions are depicted as solid lines. The propagators of $\sigma$ fields are represented by the dashed lines.

Then, the free energy density can be deduced as

$$
\begin{aligned}
\mathcal{F}_{\mathrm{OPT}}\left(T, \mu_{f}\right)= & -\frac{T}{V} \ln [\mathcal{Z}]=\Omega\left(\sigma_{l}, \sigma_{s}\right)+\mathcal{U}\left(\phi, \phi^{*}, T\right) \\
& +\Omega_{\bar{\psi} \psi}\left(T, \mu_{f}\right) .
\end{aligned}
$$

When recalling Feynman graphs up to $\delta^{2}$, the OPT approach can be depicted. Figure 1 shows the contributions in orders of $\delta$ and the color degrees of freedom $N_{c}$. The left panel (a) shows the zero order, $\delta^{0}$, with $1 / N_{c}^{0}$ or $\mathcal{O}\left(\delta^{0} N_{c}^{0}\right)$, which is shown as thick solid lines representing the fermionic contributions, for which the system is composed of quarks and antiquarks. The free energy density of these contributions have been evaluated by different approximations including MFA [12,14-21,36-38] and Hartree-Fock method (HFM) [39]. The middle panel (b) of Fig. 1 shows the first-order corrections of $\delta$ with $1 / N_{c}^{0}$ or $\mathcal{O}\left(\delta N_{c}^{0}\right)$ and how this comes up with an additional contribution rather than those of both MFA and HFM. The dashed line represents the propagator of the $\sigma$ field. This is the version we are utilizing in the present calculations.

The right panel (c) of Fig. 1 shows the second-order corrections of $\delta$ with $1 / N_{c}^{0}$ or $\mathcal{O}\left(\delta^{2} N_{c}^{0}\right)$ belonging to the nextto-leading order (NLO) expansions $[40,41]$. We notice that the first two diagrams are the propagators up to $\mathcal{O}\left(\delta^{2}\right)$. These are considered to be an OPT approach for a gas with free fermions whose masses are converged by the modified mass parameter $\eta \rightarrow \eta+\delta\left(\sigma_{f}-\eta\right)[29,35]$. The last diagram is already included in the second order of $\mathcal{O}\left(\delta^{2}\right)$ and written with the usual mass parameter $\eta[29,35]$. Let us now consider the first two diagrams to determine the PLSM free energy density in finite volume, $\mathcal{F}_{\mathrm{OPT}}\left(T, \mu_{f}\right)$, Eq. (9), the "optimized" $\delta$ expansion.

As discussed, the full construction of the OPT free energy density in finite volume is given in Eq. (9). In the right-hand side (rhs), the first term, $\Omega\left(\sigma_{l}, \sigma_{s}\right)$, stands for the potential of the mesonic contributions in pure nonstrange $\left(\sigma_{l}\right)$ and pure strange $\left(\sigma_{s}\right)$ condensates

$$
\begin{aligned}
\Omega\left(\sigma_{l}, \sigma_{s}\right)= & \left(m^{2}+(1-\delta) \eta^{2}\right) \frac{\left(\sigma_{l}^{2}+\sigma_{s}^{2}\right)}{2} \\
& +\delta\left\{\frac{\lambda_{1}}{2} \sigma_{l}^{2} \sigma_{s}^{2}+\frac{\left(2 \lambda_{1}+\lambda_{2}\right)}{8} \sigma_{l}^{4}+\frac{\left(\lambda_{1}+\lambda_{2}\right)}{4} \sigma_{s}^{4}\right. \\
& \left.-\frac{c}{2 \sqrt{2}} \sigma_{l}^{2} \sigma_{s}-h_{l} \sigma_{l}-h_{s} \sigma_{s}\right\}
\end{aligned}
$$

We now explicitly show that the last term in rhs of Eq. (9), $\Omega_{\bar{\psi} \psi}\left(T, \mu_{f}\right)$, which represents the potential of the quarks 
and antiquarks contributions and the chiral condensates in the mean-field limit reads

$$
\begin{aligned}
& \Omega_{\bar{\psi} \psi}\left(T, \mu_{f}\right) \\
& =-2 T \sum_{f=u, d, s} \int \frac{d^{3} \vec{P}}{(2 \pi)^{3}}\left[\mathcal{I}_{f}^{(+)}\left(T, \mu_{f}\right)+\mathcal{I}_{f}^{(-)}\left(T, \mu_{f}\right)\right] \\
& \quad+2 \delta N_{c} \sum_{f=u, d, s} \int \frac{d^{3} \vec{P}}{(2 \pi)^{3}}\left\{\frac{\left(m_{f}+\eta\right)}{E_{f}}\left(\eta-\sigma_{f}\right)\right. \\
& \left.\quad \times\left[1-n_{f}^{(+)}\left(T, \mu_{f}\right)-n_{f}^{(-)}\left(T, \mu_{f}\right)\right]\right\} .
\end{aligned}
$$

The Fermi-Dirac distribution functions $\mathcal{I}_{f}^{(+)}\left(T, \mu_{f}\right)$ and $n_{f}^{(+)}\left(T, \mu_{f}\right)$ are defined-in widely used notations-as

$\mathcal{I}_{f}^{(+)}\left(T, \mu_{f}\right)=\ln \left[1+3\left(\phi+\phi^{*} e^{-\frac{E_{f}^{(+)}}{T}}\right) e^{-\frac{E_{f}^{(+)}}{T}}+e^{-3 \frac{E_{f}^{(+)}}{T}}\right]$,

$$
n_{f}^{(+)}\left(T, \mu_{f}\right)=\frac{\left(\phi^{*}+2 \phi e^{-\frac{E_{f}^{(+)}}{T}}\right) e^{-\frac{E_{f}^{(+)}}{T}}+e^{-3 \frac{E_{f}^{(+)}}{T}}}{1+3\left(\phi+\phi^{*} e^{-\frac{E_{f}^{(+)}}{T}}\right) e^{-\frac{E_{f}^{(+)}}{T}}+e^{-3 \frac{E_{f}^{(+)}}{T}}},
$$

where $E_{f}^{( \pm)}=E_{f} \mp \mu_{f}$ are the energy-momentum dispersion relations, in which the upper sign is applied for quarks and the lower sign for antiquarks. These relations are subject to modifications due to OPT, $E_{f}=\left[|\vec{P}|^{2}+\left(m_{f}+\eta\right)^{2}\right]^{1 / 2}$. By replacing $E_{f}^{(+)}$with $E_{f}^{(-)}$and the order parameter of the Polyakov-loop variable $\phi$ with its conjugate $\phi^{*}$ or vice versa, we find that the terms $\mathcal{I}_{f}^{(-)}\left(T, \mu_{f}\right)$ and $n_{f}^{(-)}\left(T, \mu_{f}\right)$ are identical to $\mathcal{I}_{f}^{(+)}\left(T, \mu_{f}\right)$ and $n_{f}^{(+)}\left(T, \mu_{f}\right)$, respectively. It is worth highlighting that the second term in the rhs of Eq. (11) approximately equals the derivative of the first term in the mean-field of the averaged mass parameter $\bar{\eta}$.

The second line in Eq. (11) is approximately the gap equation of the quark condensate $[42,43]$. By minimizing the thermodynamic potential with respect to the quark condensate $\left\langle\bar{q}_{f} q_{f}\right\rangle$, this definition becomes obvious, which shall play an essential role in clarifying the impacts of OPT compared to MFA, as illustrated in Fig. 5. The modified Fermi-Dirac distribution functions for quarks and antiquarks, Eq. (13), with finite Polyakov-loop variables are derived explicitly by the summation over the Matsubara frequencies [42]. These distribution functions straightforwardly lead to the standard form, especially in the limit that $\phi, \phi^{*} \rightarrow 1$, i.e., within the deconfined phase. On the contrary, within the confined phase, $\phi, \phi^{*} \rightarrow 0$, the exponential term grows by a factor of 3 .

For the free energy density in PLSM, OPT and MFA procedures work as follows:

(i) Optimized perturbation theory (OPT): Eq. (9) is to be estimated, where Eqs. (10), (11), and (5) shall be taken into account, and

(ii) Mean-field approximation (MFA): the chiral limit of the mean-field is obtained when the thermodynamic potentials in Eq. (9) are determined at $\delta \rightarrow 1$ and $\eta=$ 0 (or vanishing arbitrary mass parameter).

Having the free energy estimated weather in OPT or in MFA, the thermodynamic quantities which are thought to describe the chiral structure of the QCD matter, at finite temperatures and finite chemical potentials can be determined. These quantities play the role of the thermodynamic order parameters characterizing whether the system undergoes phase transition. Concretely, the order parameters are the mean $\sigma$ fields $\left(\bar{\sigma}_{l}\right.$, and $\left.\bar{\sigma}_{s}\right)$ and the Polyakov-loop variables $(\bar{\phi}$, and $\left.\bar{\phi}^{*}\right)$, which are analytically estimated from the global minimizing of the real part of the PLSM free energy density in finite volume, $\mathcal{R} e\left[\mathcal{F}_{\mathrm{OPT}}(T, \mu)\right]$, with respect to the associated thermodynamic order parameter, at a saddle point

$$
\begin{aligned}
& \left.\frac{\partial \mathcal{F}_{\mathrm{OPT}}}{\partial \bar{\sigma}_{l}}\right|_{\overline{\sigma_{l}}}=0,\left.\quad \frac{\partial \mathcal{F}_{\mathrm{OPT}}}{\partial \bar{\sigma}_{s}}\right|_{\overline{\sigma_{s}}}=0,\left.\quad \frac{\partial \mathcal{F}_{\mathrm{OPT}}}{\partial \bar{\phi}}\right|_{\bar{\phi}}=0, \\
& \left.\frac{\partial \mathcal{F}_{\mathrm{OPT}}}{\partial \bar{\phi}^{*}}\right|_{\bar{\phi}^{*}}=0 .
\end{aligned}
$$

These expressions can be solved, numerically. For MFA, these expressions are the ones assuring global minimization and consequently determine the various order parameters. For OPT, we have to assure the principle of minimal sensitivity (PMS), Eq. (6), in addition to Eqs. (14). Only when all expressions outlined in Eqs. (14) and (6) are fulfilled, $T$ and $\mu$ can be determined and then enter further OPT calculations. To summarize, the differences between OPT and MFA become now obvious, namely for MFA, $\mathcal{F}_{\mathrm{OPT}} \rightarrow \mathcal{F}$ and Eq. (14) are the only global minimization to be fulfilled. For OPT, Eq. (6) must be fulfilled, as well.

In the section that follows we present results on the chiral condensates and the deconfinement order parameters, Sec. III A. Section III C is devoted to results for the thermodynamic pressure. The fluctuations and the correlations of various conserved charges shall be discussed in Sec. III D. The second-order moments shall be outlined in Sec. III D 1, while the higher-order moments are the topics of Sec. III D 2.

\section{RESULTS AND DISCUSSION}

In the present section, we study the quark-hadron phase structure of the QCD matter at finite temperature and chemical potential in MFA and OPT in PLSM. To this end, we first calculate the PLSM chiral condensates, $\sigma_{l}$ and $\sigma_{s}$, and the deconfinement order parameters $\phi$ and $\phi^{*}$. To judge about the reliability of both types of approximations, we compare the PLSM subtracted condensate for pure mesonic $\sigma$ fields with the lattice QCD (LQCD) calculations. In doing this, we can determine the pseudocritical temperatures at different chemical potentials in MFA and then in OPT. The results in both approximations shall be summarized in the QCD phase diagram compared with recent lattice QCD simulations. As a further check, we discuss some PLSM features of the thermodynamics. We limit the discussion on the pressure. Then, we move to the fluctuations and the correlations of various conserved charges, which are deduced from the second derivatives of the pressure, for instance, with respect to 


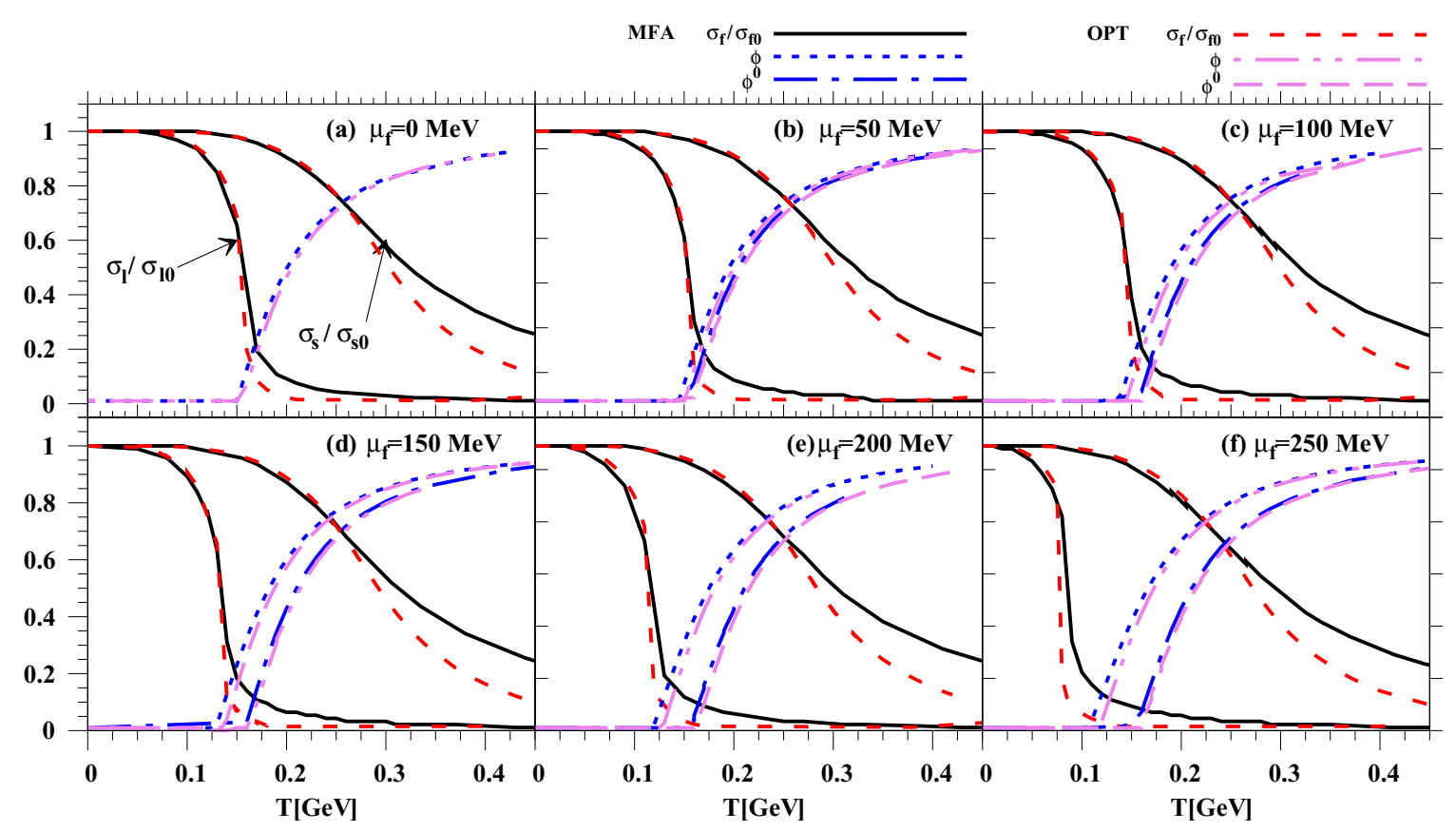

FIG. 2. (a)-(f) The temperature dependence of the normalized condensates for nonstrange $\sigma_{l} / \sigma_{l 0}$ and strange $\sigma_{s} / \sigma_{s 0}$ chiral condensates in MFA (solid curves) and in OPT (dashed curves) and that of order parameter of Polyakov-loop variables $\phi$ (dashed curves) and $\phi^{*}$ in MFA (dotted-dash curves) and $\phi$ (dotted curves) and $\phi^{*}$ in OPT (double dotted dash curves) are given at $\mu_{f}=0,50,100,150,200$, and 250 MeV, respectively.

the corresponding chemical potential. Last but not least, we compare between the PLSM results on high-order moments of particle numbers.

\section{A. Chiral condensates and deconfinement order parameters}

As discussed in Sec. II, the chiral condensates $\left(\sigma_{l}\right.$ and $\left.\sigma_{s}\right)$ and the Polyakov-loop variables $\left(\phi\right.$ and $\left.\phi^{*}\right)$ can be evaluated by solving the gap equations, Eqs. (14) and (6), $\mathcal{R} e\left[\mathcal{F}_{\mathrm{OPT}}(T, \mu)\right]$, at the subtle point. The vacuum values of nonstrange and strange chiral condensates, at vanishing chemical potential $\mu_{f}=0 \mathrm{MeV}$, are $\sigma_{l 0}=92.5 \mathrm{MeV}$ and $\sigma_{s 0}=$ 94.2 MeV, respectively. The various PLSM parameters are estimated, at $m_{\sigma}=800 \mathrm{MeV}$. As introduced, for MFA, the gap equations to be solved for the ones given in Eq. (14).

In panels (a), (b), (c), (d), (e), and (f) of Fig. 2 the temperature dependence of the order parameters which are calculated in MFA and OPT at different chemical potentials $\mu_{f}=0,50,100,150,200$, and $250 \mathrm{MeV}$, respectively, are depicted. The temperature dependence of the order parameter of the Polyakov-loop variables $\phi$ (dashed curves) and $\phi^{*}$ (dotted-dash curves) in MFA and $\phi$ (dotted curves) and $\phi^{*}$ (double dotted dash curves) and in OPT are also illustrated in the same panels, at the given chemical potentials.

Between the MFA and OPT calculations for $\phi$ and $\phi^{*}$, there are small differences below and above $T_{\chi}$. While, almost no difference exists between the PLSM calculations for $\sigma_{l}$ and $\sigma_{s}$ in MFA and OPT. In the hadronic phase, at $T<T_{\chi}$, both approximations become distinguishable, especially in the region of the phase transition. A larger difference appears in the quark-gluon phase. (i) The thermal behavior of $\sigma_{l}$ and $\sigma_{s}$ starts at almost the same value; the one corresponding to their vacuum condensates. In this region, the effect of the chemical potential is apparently negligible. The increase in chemical potential seems to repair the increase in the phase transition. With this regard, we notice that OPT is more sensitive than MFA. This is a fundamental difference which might favor OPT against MFA.

(ii) We also notice that in OPT $\sigma_{l}$ starts its prompt phase transition more rapidly than MFA. While in MFA $\sigma_{l}$ begins a likely first-order phase transition, at $\mu=$ $200 \mathrm{MeV}$, panel (e) of Fig. 2. In OPT, the first-order phase transition is indicated for $\sigma_{l}$, at $\mu=100 \mathrm{MeV}$.

(iii) For $\sigma_{s}$, there is always a smooth phase transition, although, OPT at $T \geqslant T_{\chi}$ is accompanied with a faster drop in $\sigma_{s}$ than MFA. The corresponding $T_{\chi}$ remains nearly identical, Fig. 4.

With this regard, it is in order now to summarize the procedures utilized in determining the pseudocritical temperature $T_{\chi}[20]$.

(i) The first one utilizes the temperature dependence of the chiral susceptibility. This is the second derivative with respect to the chemical potential or the first derivative of the subtracted condensate $\Delta_{l s}$, Eq. (15), with respect to the chemical potential, Eq. (17) and Fig. 6. $T_{\chi}$ is precisely positioned at the inflection point, at which a maximum in the chiral susceptibility takes place.

(ii) The second procedure utilizes the intersection point of the normalized chiral condensate and the 


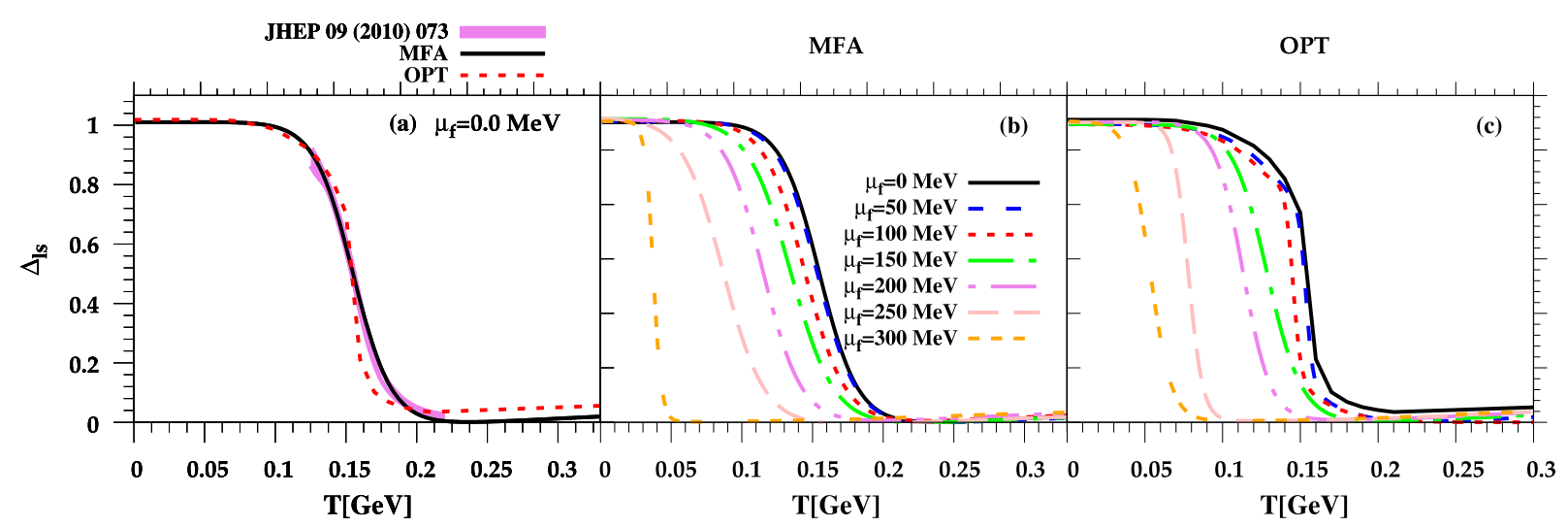

FIG. 3. Left panel (a) illustrates the temperature dependence of the subtracted condensate $\Delta_{l s}$ in MFA (solid curve) and in OPT (dashed curve) and compares these with recent lattice QCD simulations [48] (solid band). Middle panel (b) shows the MFA subtracted condensate, at $\mu=0.0$ (solid curve), 50.0 (dashed curve), 100.0 (dotted curve), 150.0 (dotted-dash curve), 200.0 (double dotted-dash curve), 250.0 , (long-dash curve), and 300.0 MeV. (dotted long-dash curve). Right panel (c) depicts the same as in the middle panel but here for OPT.

Polyakov-loop variables, as dictated by the lattice QCD simulations, Fig. 2 [44].

With this regard, we believe that the first procedure leads to a precise estimation for $T_{\chi}$, at different chemical potentials. The pseudocritical temperature $T_{\chi}$ is an essential thermodynamic quantity for the $\mathrm{QCD}$ phase structure. In the section that follows, we confront our calculations on $\sigma_{l}$ and $\sigma_{s}$ to recent lattice QCD calculations.

\section{B. Pseudocritical temperatures}

Another thermodynamic quantity which plays the role of an order parameter is the normalized net-difference between the nonstrange and strange chiral condensate, known as the subtracted condensates $\Delta_{l s}$ [45], this quantity was directly simulated in lattice QCD,

$$
\Delta_{l s}=\frac{\sigma_{l}-\left.\left(h_{l} / h_{s}\right) \sigma_{s}\right|_{T}}{\sigma_{l}-\left.\left(h_{l} / h_{s}\right) \sigma_{s}\right|_{T=0}},
$$

where $h_{l}\left(h_{s}\right)$ are nonstrange (strange) explicit symmetry breaking parameters which are to be estimated from the Dashen-Gell-Mann-Oakes-Renner (DGMOR) relations $[46,47]$. Figure 3 shows the temperature dependence of the subtracted condensate, at different chemical potentials. The left panel (a) depicts the PLSM results in MFA (solid curve) and in OPT (dashed curve). These are compared with the continuum extrapolation of recent lattice QCD simulations [48]. There is a good agreement, especially within the region of the phase transition, where the condensates decline very rapidly. It is obvious that at $T<T_{\chi}, \Delta_{l s}$ first remains unchanged having unity as value. With the increase in temperature, a rapidly decrease takes place within the region of the phase transition. With further increase in the temperature, $\Delta_{l s}$ becomes almost temperature independent. In this region, the colored quarkgluon phase, $\Delta_{l s}$ keeps its low value almost unchanged, which apparently means that the quark and gluon are deconfined and their related degrees of freedom are liberated.

The middle and right panels of Fig. 3 show the temperature dependence of the subtracted condensates at various chemical potentials for MFA and OPT, respectively. Here, we also observe that at low temperatures, $\Delta_{l s}$ keep their values (unity) unchanged for a while, i.e., until the temperatures reach some values. Again, the further increase in the temperature gives almost the same behavior as the one obtained in the left panel (a). We notice that the increase in the chemical potentials tends to increase the rate of the rapid drop in $\Delta_{l s}$. The larger chemical potential, the narrower is the temperature region, within which $\Delta_{l s}$ decline to low values. It is apparent that hadron-quark phase transition of first order seems to start taking place, at $\mu_{f}=200-250 \mathrm{MeV}$.

The results obtained when comparing $\Delta_{l s}$ with recent lattice QCD simulations, encourage mapping out the QCD phase diagram. This is another confrontation of the PLSM results in MFA and in OPT with the first-principle calculations. As discussed earlier, the pseudocritical temperatures in PLSM, $T_{\chi}$ is to be estimated in different procedures. Having this done, we can now map out $T_{\chi}$ at different baryon chemical potential $\mu_{B}=3 \mu_{f}$.

Figure 4 depicts the $T_{\chi}-\mu_{B}$ plane. The PLSM results in MFA (solid curve) and in OPT (dashed curve) are compared with the lattice QCD simulations [49] (dashed band), [50] (grid band), and also with the experimental estimations in the STAR experiment at the BNL Relativistic Heavy Ion Collider (RHIC) (closed symbols) [51] and the ALICE experiment at the CERN Large Hadron Collider (LHC) (open symbols) [52]. For the lattice QCD simulations [49], the dashed band indicates the type of the phase transition. The grid band shows the same as the dashed band but here for the first-principle lattice QCD calculations [50]. While the solid band refers to the boundary of the critical temperature, the dashed band illustrates the boundary of $T_{\chi}$, which was obtained from the baryon susceptibility, Fig. 6 .

The PLSM results in MFA (solid curve) and in OPT (dashed curve) agree well with both lattice QCD calculations. The agreement looks very convincing, especially when focusing on the lattice QCD simulations [49] (solid and dashed bands), which are recently refined [50] (grid band). The inside box zooms out the region, within which the lattice QCD calculations are reliable. When focusing on the possible differences between MFA and OPT, we would report on 


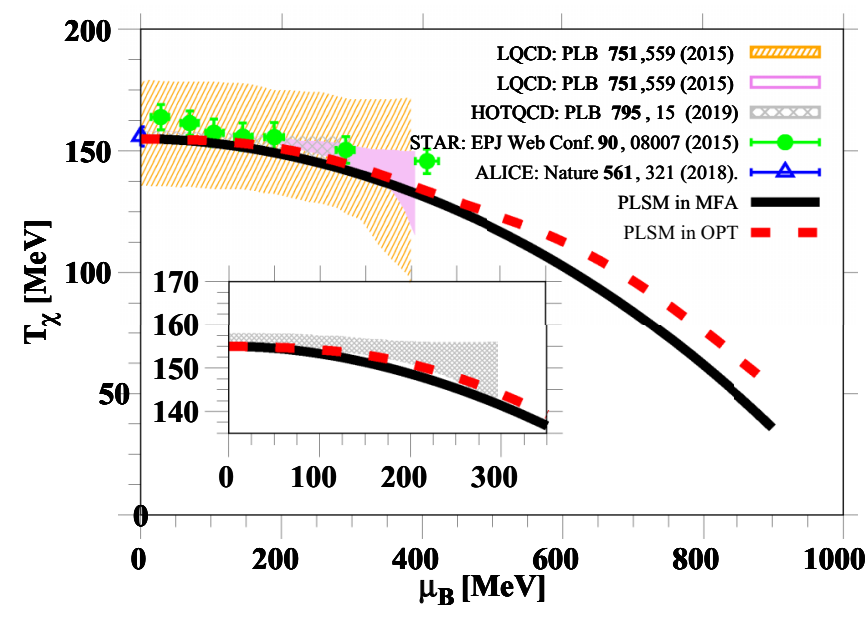

FIG. 4. The chiral phase diagram of the PLSM resulted in MFA (solid curve) and in OPT (dashed curve) is compared with recent lattice QCD simulations [49] (solid and dashed bands) and [50] (grid band). Furthermore, these are confronted to the experimental results of the STAR [51] (closed symbols) and ALICE experiments [52] (open symbols).

an almost identical result. Apart from the observation that OPT is accompanied with a slightly higher $T_{\chi}$, especially at large $\mu_{B}$, both approximations result in identical $T_{\chi}$. We notice that the phase transitions in MFA and in OPT cross over. Both result in identical $T_{\chi}$, at $\mu_{B} \lesssim 450 \mathrm{MeV}$. At larger $\mu_{B}$, they start being distinguishable; OPT has a higher $T_{\chi}$ than MFA.

So far, we conclude that either in the order parameters or in the subtracted condensates or in the pseudocritical temperatures both MFA and OPT are almost identical, especially at small $\mu_{B}$. At higher $\mu_{B}$, OPT becomes slightly more sensitive than MFA, which represents a corner milestone in preparing PLSM for an extensive scan for critical phenomena, such as QCD critical endpoint.

In the section that follows, we compare PLSM results in OPT and in MFA with recent lattice QCD calculations for the thermodynamic pressure, which can be derived directly from the total free energy density, Eq. (9).

\section{Thermodynamic pressure}

The thermodynamic pressure $p\left(T, \mu_{f}\right)=-\mathcal{F}\left(T, \mu_{f}\right)$ plays an important role in deriving various thermodynamic quantities. After addressing the PLSM in MFA and in OPT and estimating the corresponding order parameters [chiral $(\sigma$ fields) and deconfinement (Polyakov)], it is now comprehensible to analyze the thermodynamic pressure. As done in the previous section, we aim at confronting the PLSM results on the pressure with recent lattice QCD calculations [53]. The earlier have been estimated in MFA and in OPT, separately. We aim at determining which approach agrees well with the lattice QCD calculations.

With this regard, we calculate the Stefan-Boltzmann (SB) limit which can be defined from the partition function of an ideal gas of free quarks and gluons [54] with $N_{c}$ color degrees of freedom and $N_{f}$ quark degrees of freedom as

$$
\begin{aligned}
\frac{p_{\mathrm{SB}}}{T^{4}}= & \frac{0.8 G_{g}}{72} \pi^{2}+\sum_{f} \frac{G_{f}}{72}\left[0.7 \pi^{2}+3\left(\frac{\mu_{f}}{T}\right)^{2}\right. \\
& \left.+\frac{3}{2 \pi^{2}}\left(\frac{\mu_{f}}{T}\right)^{4}\right]
\end{aligned}
$$

where $G_{g}$ and $G_{f}$ are the degeneracy factors for gluons and quarks, respectively. These are defined as $G_{g}=$ spin polarization $[0,1] \times\left(N_{c}^{2}-1\right), \quad G_{f}=$ spin polarization $[+1 / 2,-1 / 2] \times \operatorname{parity}[\psi, \bar{\psi}] \times N_{c} N_{f}$, i.e., the degeneracy factors, $G_{g}=16$ and $G_{f}=36$. In this limit, i.e., taking into account the first two terms $\frac{\pi^{2}}{72}[(0.8 \times 16)+(0.7 \times 36)]$, the $\mathrm{SB}$ limit for thermodynamic pressure reads $p_{\mathrm{SB}} / T^{4}=5.209$ for $N_{f}=2+1$ quark flavors.

The upper panels of Fig. 5 depict the temperature dependence of the normalized thermodynamic pressure $p / T^{4}$ deduced form the PLSM in MFA and OPT, at different chemical potentials. The left-hand panel (a) shows the PLSM pressure in MFA and in OPT, at $\mu_{f}=0 \mathrm{MeV}$ and compares these with recent lattice QCD calculations [53] (closed circles). We observe that the PLSM results agree well with the lattice QCD calculations, as reported in Refs. [12,14-21,36]. At low temperatures, the PLSM results are slightly lower than the lattice QCD calculations. The comparison seems to be improved in the region of the quark-hadron phase transition. At high temperatures, there is a good agreement, at least up to $T \leqslant 2.5 T_{\chi}$. It is worth emphasizing that the PLSM curves - similar to the lattice QCD-seem to saturate below the SB limit. At $T \leqslant 2.5 T_{\chi}$, the gap with the SB limit is about $31.8 \%$ for MFA (solid curve) and a little bit more for OPT (dashed curve), $34.9 \%$. In light of this, one concludes that the phase transition in both approaches has the same transition (crossover), very similar to the lattice QCD calculations and the deconfined phase seems strongly correlated.

Middle (b) and right (c) panels of Fig. 5 show $p / T^{4}$ as functions of $T$, at $\mu_{f}=0,50,100,150,200,250$, and $300 \mathrm{MeV}$ in MFA and in OPT, respectively. We find that the increase in $\mu_{f}$ apparently drives the phase transition form being a smooth crossover to a prompt first-order phase transition. In light of this, the pseudocritical temperature $T_{\chi}\left(\mu_{B}\right)$ seems not to be a universal constant. At least, increasing $\mu_{B}$ decreases $T_{\chi}$, similar to the behavior depicted in Fig. 4. Not shown here, we report on other dependences, namely that $T_{\chi}$ depends - as well-on different variables, for instance, the type of the approximation used in the PLSM, the input parameters, and the Polyakov potential which is there to integrate the dynamics of gluon interaction to the PLSM chiral model.

The bottom panel of Fig. 5(d) shows differences between OPT and MFA in a semilog scale. The difference in the scaled pressure $\Delta p / T^{4}$ (double-dot-dashed line) is compared with the difference in second term of Eq. (11) (dot-dashed curve). The latter represents main contributions added by the OPT approximation, i.e., an extra term relative to MFA. We conclude that both approximations MFA and OPT seem not to be giving the same thermodynamic pressure. This would not be obvious from a blind comparison as in the top panels. 


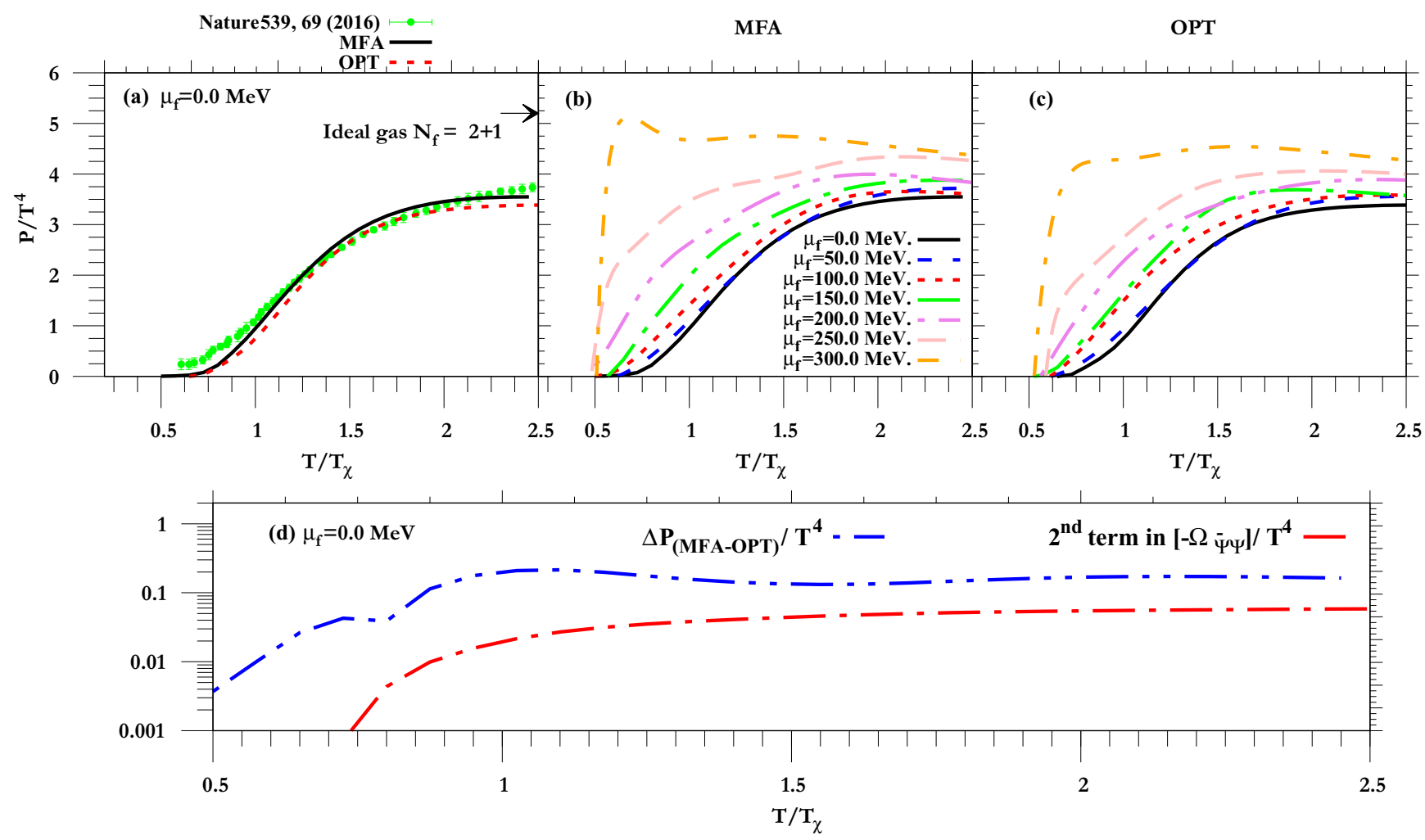

FIG. 5. The same as in Fig. 3 but here for normalized pressure $P / T^{4}$ (a)-(c). The PLSM results are compared with lattice QCD simulations [53]. The bottom panel (d) illustrates the temperature dependence of the PLSM in MFA and OPT on the normalized pressure (double-dotdashed line) and the second term in Eq. (11), the subtracted normalized pressure (dot-dashed curve).

A great portion of such a difference is to be credited to the second term of Eq. (11).

In the section that follows, we move to high-order moments. In doing this, we aim at highlighting whether the higher-order moments would enhance the difference between MFA and OPT, as observed in Fig. 5 or eventually not. We first present calculations for quadratic fluctuations for the same quantum charges and correlations, i.e., mixed quantum charges in Sec. III D 1. The higher-order moments shall be elaborated in Sec. III D 2.

\section{Fluctuations and correlations of conserved charges}

The fluctuations and the correlations of the various conserved quantum charges, $X=[B, Q, S]$, can be estimated from the derivatives of the total free energy density of the system of interest, Eq. (9), with respect to the associated chemical potential, Eq. (8),

$$
\chi_{i j k}^{\mathrm{BQS}}=\frac{\partial^{i+j+k}\left(p\left(T, \hat{\mu}_{X}\right) / T^{4}\right)}{\left(\partial \hat{\mu}_{B}\right)^{i}\left(\partial \hat{\mu}_{Q}\right)^{j}\left(\partial \hat{\mu}_{S}\right)^{k}},
$$

where $\hat{\mu}_{X}=\mu_{X} / T$, the superscripts $i, j$, and $k$ run over integers giving the orders of the derivatives. With this regard, it is informative to estimate the fluctuations (diagonal) and correlations (off-diagonal) of PLSM in MFA and in OPT. The thermal expectation values of the conserved charges $X=[B, Q, S]$, first-order moments, are estimated from the derivative of the partition function $\mathcal{Z}\left(T, \mu_{X}\right)$ with respect to corresponding chemical potential $\mu_{X}$ as

$$
\left\langle N_{X}\right\rangle=T \frac{\partial \ln \left[\mathcal{Z}\left(T, \mu_{X}\right)\right]}{\partial \mu_{X}} .
$$

The cumulants of the quantum number distributions are given as

$$
C_{n}^{X}=V T^{3} \chi_{n}^{X},
$$

where $\sigma^{2}=\left\langle\left(\delta N^{2}\right)\right\rangle=V T^{2} \chi_{2}^{X}$ is the variance and $\kappa=$ $C_{4}^{X} /\left(\sigma^{2}\right)^{2}$ is the kurtosis. One can construct products of moment, which can be related to the measured multiplicities of the produced particles [55], for instance,

$$
\kappa \sigma^{2}=\frac{C_{4}^{X}}{C_{2}^{X}}=\frac{\chi_{4}^{X}}{\chi_{2}^{X}},
$$

i.e., ratios of quartic to quadratic cumulants of the netquantum number fluctuations. The fluctuations of conserved quantum charges can be determined in the SB limit, i.e., for an ideal gas with free constituents. These are listed in Table I, where only up to the fourth order cumulants are finite. Comparing these values with our calculations indicates how far is the deconfined system from the SB limit.

In the next section, we focus on the second-order fluctuations and the correlations of various conserved charges. 
TABLE I. Cumulants for baryon, $B$, electric charge, $Q$, and strangeness quantum numbers, $S$, in an ideal gas, i.e., SB limit.

\begin{tabular}{lccc}
\hline \hline Cumulants in SB limit & $B$ & $Q$ & $S$ \\
\hline$\chi_{2}^{X}$ & $1 / 3$ & $2 / 3$ & 1 \\
$\chi_{4}^{X}$ & $2 / 9 \pi^{2}$ & $4 / 3 \pi^{2}$ & $6 / \pi^{2}$ \\
\hline \hline
\end{tabular}

\section{Second-order moments}

With this regard, the PLSM results for the second-order fluctuations are deduced at $i+j+k=2$. In the calculations presented in Fig. 6, we consider the MFA and OPT approaches, at $\mu_{f}=0$. As done in almost all figures, the PLSM results are compared with recent lattice QCD calculations.

Figure 6 depicts the temperature dependence of the quadratic fluctuations $\chi_{2}^{X}$ (left-hand panel) and the quadratic correlations $\chi_{11}^{\mathrm{XY}}$ (right-hand panel) as calculated in the PLSM in MFA (solid curves) and in OPT (dashed curves) as functions of $T$, at vanishing chemical potential. Our calculations, the diagonal fluctuations, $\chi_{2}^{X}$, are compared with recent lattice QCD calculations; Wuppertal-Budapest (grid-filled bands) [56] and HotQCD [57] (solid-filled bands) and [58] (solid curve). Figure 6 shows in panels (a), (b), and (c) the susceptibilities for the net-baryon numbers, the net-electric charge, and the net-strangeness, respectively. The phase transition (crossover) is defined where the mesonic degrees of freedom start to be liberated from the confined phase (bound mesons) and the system is converted to the deconfined (free quarks and gluons) phase [59,60].

It is obvious that the susceptibilities seem to have small values, at low temperatures, where in this region the mesonic contributions become dominant. Accordingly, the chiral condensates have maximum values due to the relevant degrees of freedom responsible for the small fluctuations, Fig. 2. It is apparent that in the region of crossover the fluctuations rapidly arise with the increase in temperature. It should be noticed that the chiral structure of the mesonic states plays an essential role in the temperature dependence of the fluctuations and the quark number multiplicities. It is believed that a new state of matter, i.e., massless quark and gluons, is produced.

At high temperatures, the system reaches the deconfined state and therefore the fluctuations raise from fixed values to maxima, even if remains slightly below the SB limit. The explicit calculations from PLSM, as well, reach $\approx 94.3 \%$ and $\approx 88.8 \%$ of their respective ideal gas limits in MFA and in
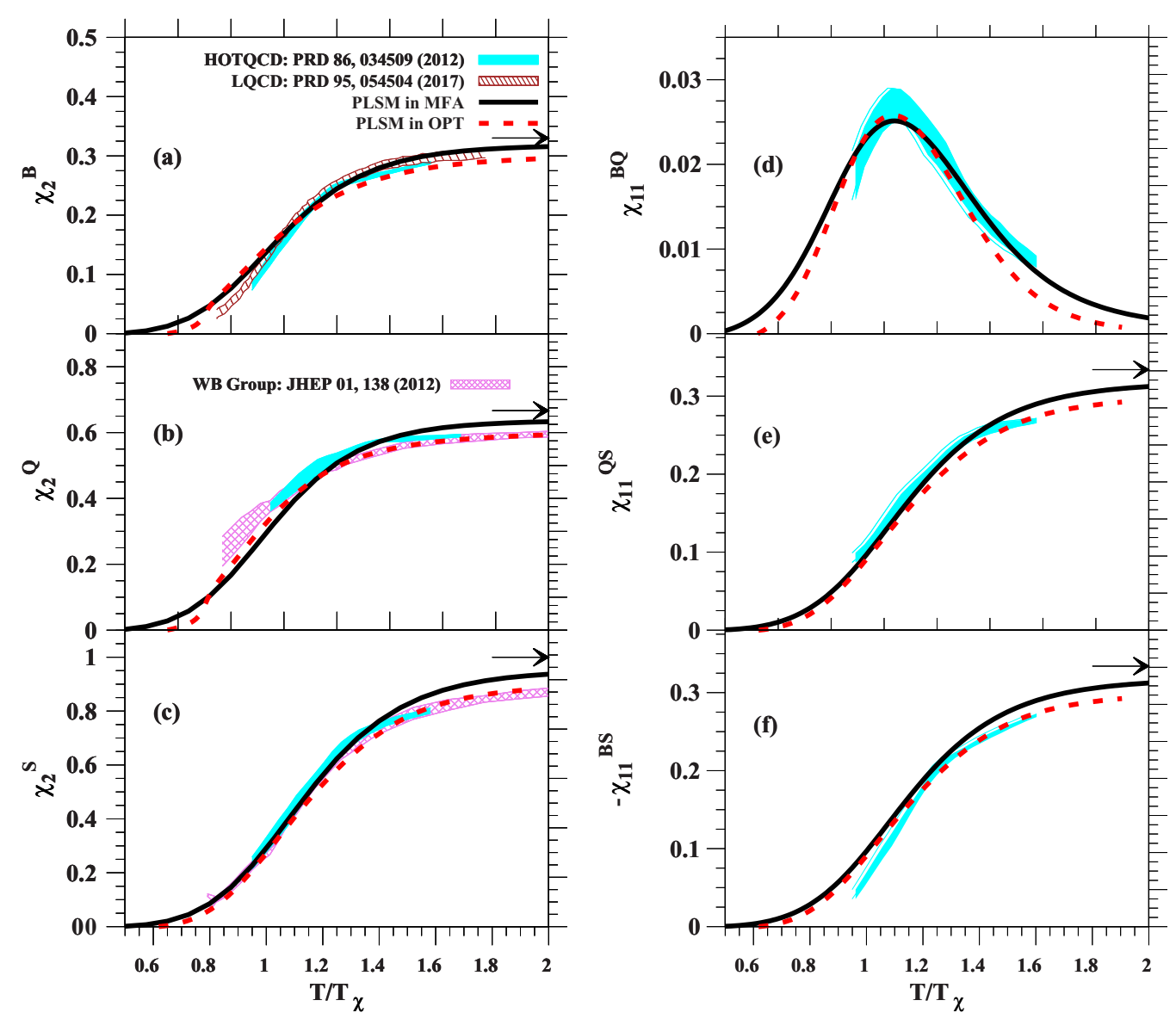

FIG. 6. Left-hand panels illustrate the temperature dependence of the second-order fluctuations of the conserved charges $\chi_{2}^{B}, \chi_{2}^{Q}$, and $\chi_{2}^{S}$ in (a), (b), and (c), respectively. The PLSM results in MFA (solid curve) and in OPT (dashed curve) are confronted to recent lattice QCD calculations [56] (grid bands), [57] (solid bands), and [58] (dash band). Right-hand panels show the same as in the left-hand panels but here for the correlations of the conserved charges $\chi_{11}^{\mathrm{BQ}}, \chi_{11}^{\mathrm{QS}}$, and $\chi_{11}^{\mathrm{BS}}$ in $(\mathrm{d}),(\mathrm{e}),(\mathrm{f})$, respectively. 

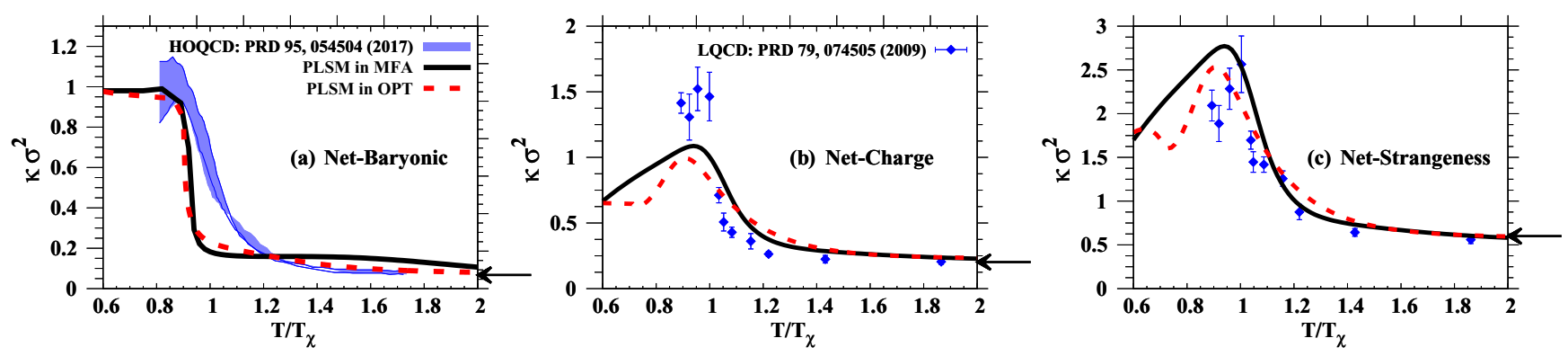

FIG. 7. The temperature dependence of the moment products $\kappa \sigma^{2}$ computed in PLSM in MFA (solid curve) and in OPT (dashed curve) for net-baryon (a), net-charge (b), and net-strangeness (c) multiplicities are compared with the lattice QCD calculations by HotQCD collaborations (solid band) [58] and [60] (closed symbols). The arrows refers to the respective SB limits.

OPT, respectively. We notice that the differences between the PLSM results and the corresponding SB limits vary according to different scenarios, for instance, the several mechanisms of the inclusion dynamics and the degrees of freedom of quark flavors and gluons. Furthermore, the differentiation with respect to $\mu_{f}$ and the various types of statistical errors in the numerical simulations would contribute to the potential differences.

The right-hand panel of Fig. 6 illustrates the same as in the left-hand panel but here for the off-diagonal fluctuations (correlations) of the conserved quantum charges $\chi_{11}^{\mathrm{XY}} ; \chi_{11}^{\mathrm{BQ}}$ (top panel), $\chi_{11}^{\mathrm{QS}}$ (middle panel), and $-\chi_{11}^{\mathrm{BS}}$ (bottom panel). We notice that the PLSM correlations agree well with the lattice QCD calculations with continuum extrapolation [57]. The variation in the temperature behavior of the correlations of the baryon number with the electric charge, $\chi_{11}^{\mathrm{BQ}}$, panel (d), is dominated by the mesonic contributions, at low temperatures. $\chi_{11}^{\mathrm{BQ}}$ arises almost exponentially with the increase in the temperature. Again $\chi_{11}^{\mathrm{BQ}}$ vanishes, at high temperatures, because in this limit the quarks become massless $[59,60]$. While the correlations of the strangeness $S$ with the electric charge $Q$ and that of the strangeness $S$ with the baryon number $B$; $\chi_{11}^{\mathrm{QS}}$ and $-\chi_{11}^{\mathrm{BS}}$, respectively, clearly manifest the effect of the strangeness degrees of freedom $[61,62]$. These correlations are related to the quark-flavor fluctuations: $\chi_{11}^{\mathrm{BS}}=-\left(\chi_{2}^{s}+\right.$ $\left.2 \chi_{11}^{u s}\right) / 3$ and $\chi_{11}^{\mathrm{QS}}=\left(\chi_{2}^{s}-\chi_{11}^{u s}\right) / 3$ [57]. As emphasized when discussing the quadratic fluctuations, the correlations here are also sensitive to the quark-hadron contributions. We observe that the PLSM results agree well with the continuum extrapolation of the lattice QCD calculations, especially at low temperature and within the region of crossover. At high temperatures, we find that the PLSM results on $\chi_{11}^{\mathrm{QS}}$ and $-\chi_{11}^{\mathrm{BS}}$ are below the SB limit. Such a difference is to be estimated as $\approx 6 \%$ and $\approx 12 \%$ for MFA and OPT, respectively.

We conclude that the comparison between the PLSM results and the lattice QCD calculations shows that OPT reproduces the first-principle fluctuations relatively better than MFA, especially within the region of the phase transition. Also, OPT seems to reproduce well the available lattice QCD correlations better that MFA.

\section{Higher-order moments}

The ratios of the higher-order moments, such as $\chi_{4}^{B} / \chi_{2}^{B}$, $\chi_{4}^{Q} / \chi_{2}^{Q}, \chi_{4}^{S} / \chi_{2}^{S}, \chi_{6}^{B} / \chi_{2}^{B}$, and $\chi_{8}^{B}$, are proposed as experimental signatures for the phase transition [63]. The PLSM results on the higher-order moments of different quantum charges in MFA are compared with the ones in OPT. We also compare these with the lattice QCD simulations and the available experimental results. We start first with the PLSM results on the product of the higher-order moments $\kappa \sigma^{2}$ or $\chi_{4}^{X} / \chi_{2}^{X}$, Eq. (20), for which measurements at various beam energies, for instance Ref. [64], and the lattice QCD calculations, at finite temperatures $[58,60]$ are available, Fig. 7.

The susceptibility of the net-baryon number is proposed as a signature for deconfining hadrons into colored massless quarks and gluons [65]. It was suggested that the particle number correlations are directly coupled to the variance of the order parameter and thus becomes sensitive to the correlation length $\xi$ in a universal manner that $\chi_{n} \sim \xi^{n(5-\eta) / 2-3}$, where $\eta$ is a critical exponent to be defined from the universality class. With this regard, we highlight that the higher-order moments of conserved charges, which are proportional to powers of the correlation length and expected to diverge at the critical endpoint of the QCD phase boundary, play an essential role in locating CEP and thus providing an important signature for its experimental characteristics.

In Fig. 7, $\kappa \sigma^{2}$ for net-baryon number (left panel), for electric charge (middle panel), and for strangeness (right panel) are depicted as functions of $T$, at $\mu_{B}=0 \mathrm{MeV}$. The results are compared with the lattice QCD calculations [60] (closed symbols) and HotQCD collaboration [58]. We find that at low temperatures $\chi_{4}^{B} / \chi_{2}^{B}$ starts from unity, where the mesonic contributions are dominant within the hadron phase. With the increase in $T$, a rapid drop in $\chi_{4}^{B} / \chi_{2}^{B}=\kappa \sigma^{2}$ takes place, due to the rapid increase in $\chi_{2}^{B}$ within the region of crossover, left panel of Fig. 7. At high temperatures, the temperature dependence of $\chi_{4}^{B} / \chi_{2}^{B}$ reaches a saturated plateau, even with much lower values than that in the confined phase. We notice that the PLSM results in MFA and OPT seem to nearly approach the SB limit, $\chi_{4}^{B} /\left.\chi_{2}^{B}\right|_{S B}=2 /\left(3 \pi^{2}\right)$.

Middle and right-hand panels of Fig. 7 draw the moment product $\kappa \sigma^{2}$ for the net-electric charge and the net-strangeness multiplicity, respectively, as calculated from the PLSM and compared with the lattice QCD calculations (closed symbols) [60]. It is apparent that the $\kappa \sigma^{2}$ approaches unity, at low temperatures and also the SB limit, at high temperatures, while at $T \sim T_{\chi}$, there is an obvious peak observed in both quantum charges [60]. At higher temperatures, $\kappa \sigma^{2}$ for the netcharge agrees with the lattice QCD simulations, while that of 

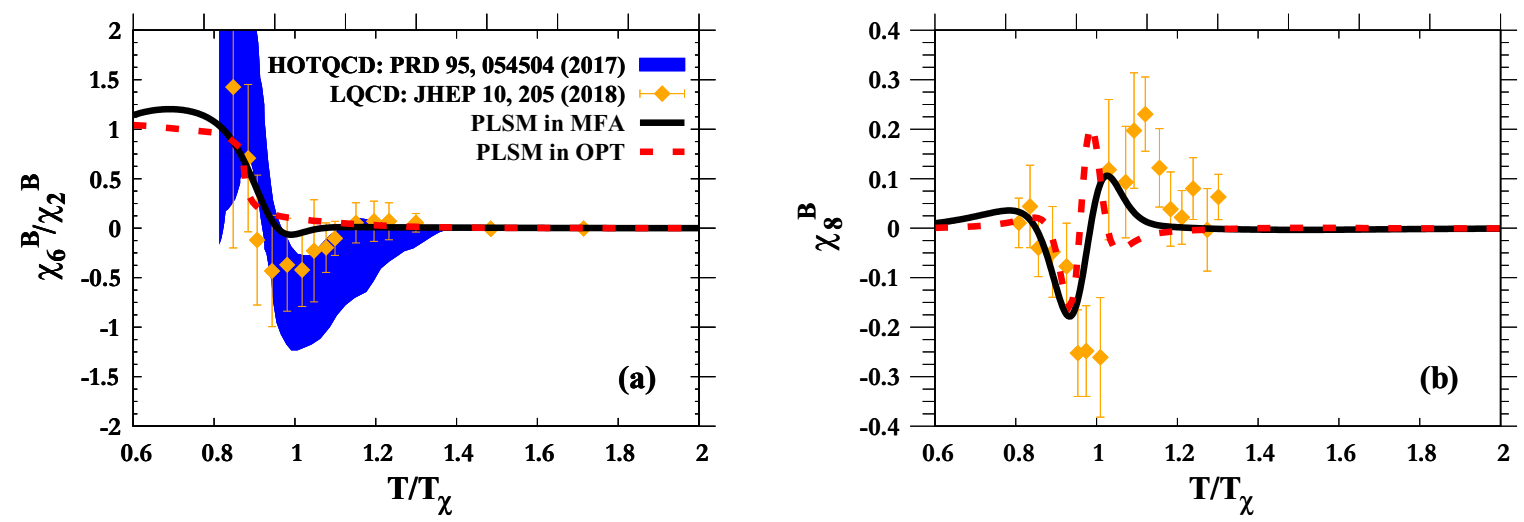

FIG. 8. The temperature dependence of susceptibilities of the conserved charges $\chi_{6}^{B} / \chi_{2}^{B}$ and $\chi_{8}^{B}$ computed in PLSM in MFA (solid curve) and in OPT (dashed curve) is graphically compared with the lattice QCD calculations [66] (solid symbols) and [58] (bands).

net-strangeness multiplicity seems to have a different dependence, at least within the region of temperatures covered by the lattice calculations we compare with. These lattice calculations are about 10 years old. To the authors' best knowledge, so far no recent calculations are available to compare with. We observe a qualitative agreement, especially at high temperatures so that the results approach the SB limits $2 / \pi^{2}$ and $6 / \pi^{2}$ for the net-electric charge and the net-strangeness multiplicities, respectively.

The comparison between the PLSM results in MFA and in OPT is qualitatively possible when confronting both results with the continuum extrapolation of the lattice QCD results [58]. In Fig. 8, the temperature dependence of the susceptibilities of the conserved charges $\chi_{6}^{B} / \chi_{2}^{B}$ (left panel) and $\chi_{8}^{B}$ (right panel) computed in PLSM in MFA (solid curve) and in OPT (dashed curve) is compared with the lattice QCD calculations [66] (solid symbols) and [58] (bands).

For $\chi_{6}^{B} / \chi_{2}^{B}$ (left panel), at $T / T_{\chi} \gtrsim 1.2$, although with the vanishing temperature dependence, the PLSM results agree excellently with the lattice QCD calculations. At lower temperatures, we find a minimum at $T_{\chi}$ followed by a rapid arise. This continues to a maximum at $\chi_{4}^{B} / \chi_{2}^{B} \approx 1$. The lattice QCD calculations are limited to $0.8 T_{\chi}$. The temperature dependence of these quantities are significant for the quark-hadron phase transition $[59,60]$. Within their large error bars, we conclude that both PLSM approaches, MFA and OPT, at least qualitatively, reproduce well the recent lattice QCD calculations.

For $\chi_{8}^{B}$ (right panel), the excellent agreement, at $T / T_{\chi} \gtrsim$ 1.2 exists here, as well. Decreasing the temperature unveils an interesting structure. It seems that a sinusoidal dependence is obtained. The sinusoidal oscillation is also supported by the lattice QCD calculations [66]. The most remarkable features of such PLSM results in both MFA and OPT likely indicate a smooth crossover between the hadronic and partonic phases. At $T / T_{\chi} \lesssim 0.8, \chi_{8}^{B}$ vanishes. The structure produced by OPT fits well with the lattice QCD simulations.

So far, we conclude that OPT is more sensitive to the higher-order moment products $\chi_{6}^{B} / \chi_{2}^{B}$ and $\chi_{8}^{B}$ than the MFA. The off-diagonal cumulants of the net-proton, the net-charge, and the net-kaon multiplicity distributions are also measured in the STAR experiment, at energies ranging between 7.7 and $200 \mathrm{GeV}$ within the rapidity $|y|<0.5$ and the transverse momentum range $0.4<p_{T}<2.0 \mathrm{GeV}$ [67-69]. In a future study, a comprehensive comparison with the STAR results is intended.

\section{CONCLUSIONS}

We have generalized PLSM calculations in order to incorporate zero- and higher-order $\delta$ expansions, i.e., MFA and OPT. While MFA is originated in statistical physics and was applied to a wide spectrum of numerical implications [12,14$21,36]$, OPT was developed in $\mathcal{O}(N) \phi^{4}$ theory in order to resume the higher-order terms of the naive perturbation approach. In MFA the time evolution of fields are averaged at a fixed time, while in OPT the time evolution of fields are estimated, perturbatively. We have intended to point out whether the MFA generalization, OPT, reproduces identical results or eventually improves the PLSM calculations as reported in MFA. The present article is the first which introduces a comprehensive comparison between MFA and OPT. If improvements are achieved, we aim at highlighting how the high-order moments are affected by OPT.

At finite temperatures and chemical potentials, we have compared the chiral condensates and the decofinement order parameters, the thermodynamic pressure, the subtracted condensates, the pseudocritical temperatures, the second- and high-order moments of various conserved charges (cumulants) obtained in MFA with the ones obtained in OPT. These calculations are then confronted to available lattice QCD simulations. In general, we conclude that when moving to lower- to higher-order moments, the OPT approach becomes more and more closer to lattice QCD simulations than the MFA. Concretely, we have found that OPT is more sensitive to $\chi_{6}^{B} / \chi_{2}^{B}$ and $\chi_{8}^{B}$ than the MFA. The higher-order moments of conserved charges is conjectured to play an essential role in positioning CEP. That OPT is found slightly more sensitive than MFA furnishes PLSM with slight more capability to predict signature for CEP and its possible experimental detection.

The convincing OPT results outlined in this study encourage the trial to compare with the measurements that are and shall be available in the near future. Accordingly, some PLSM 
parameters can be adjusted, properly, on one hand. On the other hand, the critical phenomena that might be detected in the experimental results and are sensitive the higher-order moments could likely be predicted.

\section{ACKNOWLEDGMENTS}

The work of A.T. was supported by the ExtreMe Matter Institute (EMMI) at the GSI Helmholtz Centre for Heavy Ion Research, visiting researcher.
[1] J. S. Schwinger, Phys. Rev. 82, 914 (1951).

[2] J. S. Schwinger, Phys. Rev. 91, 713 (1953).

[3] J. Schwinger, Phys. Rev. 91, 728 (1953).

[4] J. Schwinger, Phys. Rev. 92, 1283 (1953).

[5] J. Schwinger, Phys. Rev. 93, 615 (1954).

[6] J. Schwinger, Phys. Rev. 94, 1362 (1954).

[7] M. Gell-Mann and M. Levy, Nuovo Cim. 16, 705 (1960).

[8] J. S. Schwinger, Ann. Phys. (NY) 2, 407 (1957).

[9] M. C. Birse, J. Phys. G 20, 1537 (1994).

[10] D. Roder, J. Ruppert, and D. H. Rischke, Phys. Rev. D 68 , 016003 (2003).

[11] S. Gallas, F. Giacosa, and D. H. Rischke, Phys. Rev. D 82, 014004 (2010).

[12] A. N. Tawfik and A. M. Diab, Phys. Rev. C 91, 015204 (2015).

[13] C. Wesp, H. van Hees, A. Meistrenko, and C. Greiner, Eur. Phys. J. A 54, 24 (2018).

[14] A. N. Tawfik, A. M. Diab, and M. T. Hussein, J. Phys. G 45, 055008 (2018).

[15] A. M. Abdel Aal Diab and A. N. Tawfik, EPJ Web Conf. 177, 09005 (2018).

[16] A. N. Tawfik, A. M. Diab, and M. T. Hussein, Chin. Phys. C 43, 034103 (2019).

[17] A. N. Tawfik, A. M. Diab, N. Ezzelarab, and A. G. Shalaby, Adv. High Energy Phys. 2016, 1381479 (2016).

[18] A. N. Tawfik, A. M. Diab, and T. M. Hussein, Int. J. Adv. Res. Phys. Sci. 3, 4 (2016).

[19] A. N. Tawfik, A. M. Diab, and M. T. Hussein, J. Exp. Theor. Phys. 126, 620 (2018).

[20] A. N. Tawfik, A. M. Diab, and M. T. Hussein, Int. J. Mod. Phys. A 31, 1650175 (2016).

[21] A. N. Tawfik, A. M. Diab, M. T. Ghoneim, and H. Anwer, Int. J. Mod. Phys. A 34, 1950199 (2019).

[22] J. Randrup, Nucl. Phys. A 616, 531 (1997).

[23] T. Kunihiro and T. Hatsuda, Prog. Theor. Phys. 71, 1332 (1984).

[24] M. H. Thoma and H. J. Mang, Z. Phys. C 44, 349 (1989).

[25] M. H. Thoma, Z. Phys. C 44, 343 (1989).

[26] R. L. S. Farias, G. Krein, and R. O. Ramos, Phys. Rev. D 78, 065046 (2008).

[27] D. C. Duarte, R. L. S. Farias, P. H. A. Manso, and R. O. Ramos, Phys. Rev. D 96, 056009 (2017).

[28] D. S. Rosa, R. L. S. Farias, and R. O. Ramos, Physica A 464, 11 (2016).

[29] T. E. Restrepo, J. C. Macias, M. B. Pinto, and G. N. Ferrari, Phys. Rev. D 91, 065017 (2015).

[30] S. Weinberg, The Quantum Theory of Fields, Vol. 1: Foundations (Cambridge University Press, Cambridge, 2005).

[31] B.-J. Schaefer and M. Wagner, Phys. Rev. D 79, 014018 (2009).

[32] J. T. Lenaghan, D. H. Rischke, and J. Schaffner-Bielich, Phys. Rev. D 62, 085008 (2000).

[33] P. M. Stevenson, Phys. Rev. D 23, 2916 (1981).

[34] P. M. Stevenson, Phys. Rev. D 24, 1622 (1981).
[35] J.-L. Kneur, M. B. Pinto, and R. O. Ramos, Phys. Rev. C 81, 065205 (2010).

[36] A. M. Abdel Aal Diab, A. N. Tawfik, and M. T. Hussein, Electromagnetic effects on strongly interacting QCD-Matter, arXiv:1611.06926 (2016).

[37] B.-J. Schaefer, M. Wagner, and J. Wambach, Phys. Rev. D 81, 074013 (2010).

[38] H. Mao, J. Jin, and M. Huang, J. Phys. G 37, 035001 (2010).

[39] S. P. Klevansky, Rev. Mod. Phys. 64, 649 (1992).

[40] V. Dmitrasinovic, H. J. Schulze, R. Tegen, and R. H. Lemmer, Ann. Phys. (NY) 238, 332 (1995).

[41] M. Oertel, M. Buballa, and J. Wambach, Phys. Lett. B 477, 77 (2000).

[42] H. Hansen, W. M. Alberico, A. Beraudo, A. Molinari, M. Nardi, and C. Ratti, Phys. Rev. D 75, 065004 (2007).

[43] P. Costa, M. C. Ruivo, C. A. de Sousa, H. Hansen, and W. M. Alberico, Phys. Rev. D 79, 116003 (2009).

[44] P. Cea, L. Cosmai, and M. D'Elia, Nucl. Phys. B - Proc. Suppl. 129, 751 (2004).

[45] L. M. Haas, R. Stiele, J. Braun, J. M. Pawlowski, and J. Schaffner-Bielich, Phys. Rev. D 87, 076004 (2013).

[46] M. Gell-Mann, R. J. Oakes, and B. Renner, Phys. Rev. 175, 2195 (1968).

[47] R. F. Dashen, Phys. Rev. 183, 1245 (1969).

[48] S. Borsanyi et al., J. High Energy Phys. 09 (2010) 073.

[49] R. Bellwied et al., Phys. Lett. B 751, 559 (2015).

[50] A. Bazavov et al., Phys. Lett. B 795, 15 (2019).

[51] S. Das, EPJ Web Conf. 90, 08007 (2015).

[52] A. Andronic, P. Braun-Munzinger, K. Redlich, and J. Stachel, Nature 561, 321 (2018).

[53] S. Borsanyi et al., Nature 539, 69 (2016).

[54] J. I. Kapusta and C. Gale, Finite-Temperature Field Theory: Principles and Applications (Cambridge University Press, Cambridge, 2006).

[55] X. Luo and N. Xu, Nucl. Sci. Tech. 28, 112 (2017).

[56] S. Borsanyi et al., J. High Energy Phys. 01 (2012) 138.

[57] A. Bazavov et al., Phys. Rev. D 86, 034509 (2012).

[58] A. Bazavov et al., Phys. Rev. D 95, 054504 (2017).

[59] S. Borsanyi et al., J. Phys. G 38, 124060 (2011).

[60] M. Cheng et al., Phys. Rev. D 79, 074505 (2009).

[61] V. Koch, A. Majumder, and J. Randrup, Phys. Rev. Lett. 95, 182301 (2005).

[62] S. Jeon and V. Koch, Phys. Rev. Lett. 85, 2076 (2000).

[63] B. Friman, F. Karsch, K. Redlich, and V. Skokov, Eur. Phys. J. C 71, 1694 (2011).

[64] L. Adamczyk et al., Phys. Lett. B 785, 551 (2018).

[65] M. A. Stephanov, Phys. Rev. Lett. 102, 032301 (2009).

[66] S. Borsanyi et al., J. High Energy Phys. 10 (2018) 205.

[67] R. Esha, Nucl. Phys. A 967, 457 (2017).

[68] R. Esha, PoS CPOD2017, 003 (2018).

[69] L. Chen, Z. Li, F. Cui, and Y. Wu, Nucl. Phys. A 957, 60 (2017). 